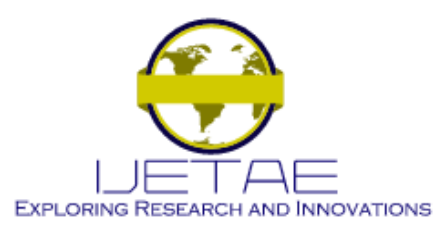

International Journal of Emerging Technology and Advanced Engineering

Website: www.ijetae.com (ISSN 2250-2459, ISO 9001:2008 Certified Journal, Volume 10, Issue 10, October 2020)

\title{
Coulomb Potential as Metrics of Hyperbolic Space
}

\author{
Yamaleev Robert M. \\ Joint Institute for Nuclear Research, Laboratory of Informational Technology \& Dubna, Russia.
}

\begin{abstract}
Representation of the Coulomb field as a metrics of the hyperbolic space within the Beltrami- Poincare model of hyperbolic geometry is done. In order to connect the potential of the electric field with the length of a geodesic line a new characteristics of the Coulomb field, the entropy, is introduced.
\end{abstract}

Keywords - Entropy, Coulomb's law, hyperbolic space, Poincare-Beltrami model, potential field.

\section{INTRODUCTION}

The similarity of the Coulomb potential for a point charge [1] with the hyperbolic space metric in the Beltrami - Poincare model [2], given in the horo-cyclic coordinate system [3], leads to the idea of a possible connection between the Coulomb field potential and the hyperbolic space metric. This paper is devoted to the implementation of this idea and consists of the following parts. In the first part, we introduce a new integral characteristic of the field - a definite integral of the potential of a single electric field. Further, we consider separately the potential determined by the work of the field performed on the test charge when it moves in the field from a given point to infinity and the potential determined by the work of the field perfect from one point of the field to another, located at a finite distance. In the latter case, we are dealing with a difference of potentials. The definite integral of the Coulomb potential has properties similar to entropy in thermodynamics, for that reason, this characteristics of the field we propose to call as the field entropy. Its geometric meaning corresponds to the length of the geodesic line in the Beltrami-Poincare model of the Lobachevsky hyperbolic plane.

In the second part, we outline the model of the BeltramiPoincare model of the hyperbolic plane, the definition of the length of the geodesic line in which gives an adequate geometric description of the Coulomb field.

\section{Integral Of The Coulomb Field Potential AND THE CROSS-RATIO}

The potential of a (potential) field is defined as the work of the field on the test charge when the latter is moved between two points of a given curve inside the space of the field's action on the charge.
The area of absence of the field action is a single space for all test charges, which allows us to introduce the concept of the potential of a given point in space. However, within the action of the field, the field potential depends on two variables - the coordinate of the beginning and the coordinate of the end of the line along which the work on the test charge was performed. Clarification of these circumstances is important to determine the integral characteristic of the field.

The usual definition of the field potential assumes that the action of the field at infinity is zero therefore the potential is given by a function of one variable-the radial distance from the charge

$$
U(r)=k \int_{\infty}^{r} \frac{d x}{x^{2}}=k \frac{1}{r}, k=\frac{e^{2}}{4 \pi \varepsilon} .
$$

The definite integral of the potential function has the form of a logarithmic function

$$
S_{a b}=k \int_{b}^{a} \frac{1}{r} d r=k \log \frac{a}{b}
$$

Now, let's consider the case when the field potential depends on two variables - the coordinates of the beginning and the end of the line along which the work on the test charge is performed. This function is defined as the difference between two potentials (voltage) defined at different distances

$$
U_{1}-U_{2}=k \int_{2}^{1} \frac{d x}{x^{2}}=k\left(\frac{1}{r_{1}}-\frac{1}{r_{2}}\right) .
$$

For our purposes, this will override by the formula in the following way

$$
U_{12}(r)=U\left(r_{1}-r\right)-U\left(r_{2}-r\right) .
$$

The form of the function (3) coincides with the potential of the dipole, so the definition given by the formula (4) is called the dipolar potential. It is seen, the dipolar potential depends on one variable and two constants. The integral on the dipolar potential is given by the difference of logarithm functions 


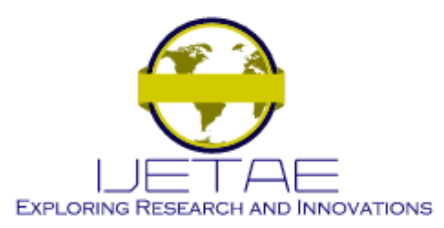

International Journal of Emerging Technology and Advanced Engineering

Website: www.ijetae.com (ISSN 2250-2459, ISO 9001:2008 Certified Journal, Volume 10, Issue 10, October 2020)

$$
\phi_{a}-\phi_{b}=\int_{b}^{a} U_{12}(r) d r=k\left(\log \frac{x_{1}-a}{x_{1}-b}-\log \frac{x_{2}-a}{x_{2}-b}\right)
$$

Next, let us present (5) in the exponential form

$$
\exp \left(\frac{1}{k}\left(\phi_{a}-\phi_{b}\right)\right)=\frac{a-x_{1}}{a-x_{2}} \frac{b-x_{2}}{b-x_{1}} .
$$

The right-hand side of the equation (6) has the form of a cross-ratio.

Let us establish a connection between definitions (2) and (5). To this aim, let us write equation (2) in the exponential form

$$
\exp \left(\frac{1}{k} S_{12}\right)=\frac{x_{1}}{x_{2}}
$$

Simultaneous translation of the numerator and denominator of the fraction results in translation of the variable on the exponent

$$
\exp \left(\frac{1}{k} S_{12}+\phi_{a}\right)=\frac{x_{1}-a}{x_{2}-a}
$$

The translation in the opposite direction for the other parameter of translation gives

$$
\exp \left(-\frac{1}{k}\left(S_{12}+\phi_{b}\right)\right)=\frac{x_{2}-b}{x_{1}-b}
$$

The product of these two exponents leads to the crossratio presented in (6).

\section{Beltrami- PoInCARE MOdel Of THE LOBACHEVSKY HYPERBOLIC PLANE}

The square of the interval in the Beltrami - Poincare model of the Lobachevsky hyperbolic plane defined in horo-cyclic coordinates has the form [3]

$$
d s^{2}=k \frac{d \xi^{2}+d \eta^{2}}{\eta^{2}}
$$

Now, let us seek an equation of the geodesic line. Since the metrics (10) does not depend on the coordinate $\xi$, it is convenient to search for the equation of a straight line as follows. The line length is given by the integral

$$
S=\int L d \eta, \quad L=k \frac{1}{\eta} \sqrt{1+\dot{\xi}^{2}}
$$

A straight line, being the shortest one, is given by the Lagrange equation

$$
\frac{d}{d \eta} \frac{\partial L}{\partial \dot{\xi}}-\frac{\partial L}{\partial \xi}=0
$$

which immediately integrated

$$
\frac{\dot{\xi}}{\eta \sqrt{1+\dot{\xi}^{2}}}=C,
$$

where $\mathrm{C}$ is the integration constant. If $C=0$, then,

$$
\xi\left(\eta_{1}\right)=\xi\left(\eta_{2}\right)
$$

This case corresponds to vertical straight lines. The distance along this line is determined by the formula

$$
S_{12}=k\left(\log \eta_{1}-\log \eta_{2}\right) \text {. }
$$

Notice, this formula coincides with the formula defined in (2) for the field entropy of the electrostatic field of a single charge, therefore, this characteristic of the field receives a geometric interpretation as the length of a geodesic line, where $\mathrm{k}$ is a constant characteristic of this space.

Now consider the case $C \neq 0$. Denote $C=\frac{1}{R}$ and solve equation (13) with respect to $\xi$, we obtain

$$
\frac{d \xi}{d \eta}= \pm \frac{\eta}{\sqrt{R^{2}-\eta^{2}}}
$$

The integration of this equation leads to the equation for a circle

$$
R^{2}=\left(\xi-\xi_{0}\right)^{2}+\eta^{2}
$$

This equation is also equation for the geodesic line (straight line) on the Beltrami-Poincare half-plane. To derive a formula for the distance between points $\left(\xi_{1}, \eta_{1}\right)$ and $\left(\xi_{2}, \eta_{2}\right)$ write the circle equation (17) in the parametric form

$$
\xi=\xi_{0}+R \cos \theta, \eta=R \sin \theta .
$$




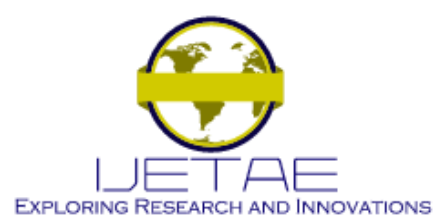

International Journal of Emerging Technology and Advanced Engineering

Website: www.ijetae.com (ISSN 2250-2459, ISO 9001:2008 Certified Journal, Volume 10, Issue 10, October 2020)

According to formulae (10) and (11), the length of this line is determined by the formula

$$
S_{12}=k \int_{2}^{1} \frac{d \theta}{\sin \theta}=k\left(\log \tan \frac{\theta_{1}}{2}-\log \tan \frac{\theta_{2}}{2}\right) .
$$

This formula also is read as

$$
\exp \left(S_{12} / k\right)=\tan \frac{\theta_{1}}{2} / \tan \frac{\theta_{2}}{2} .
$$

In order to express the length in the variables $\left(\xi_{1}, \eta_{1}\right)$ and $\left(\xi_{2}, \eta_{2}\right)$, we have to perform a number of trigonometric transformations. To this aim, let us derive the formula for the cosine of length

$$
\cosh \left(S_{12} / k\right)=\frac{1}{2}\left(\exp \left(\frac{S_{12}}{k}\right)+\exp \left(-\frac{S_{12}}{k}\right)\right) \text {. }
$$

Now, let us use the formula $\tan ^{2} \frac{B}{2}=\frac{1+\cos B}{1-\cos B}$ and definitions (18). Substitution (20) into (21) gives

$$
\begin{aligned}
& \cosh \left(S_{12} / k\right)=\frac{1}{2} \frac{\tan ^{2}\left(\theta_{1} / 2\right)+\tan ^{2}\left(\theta_{2} / 2\right)}{\tan \left(\theta_{1} / 2\right) \tan \left(\theta_{2} / 2\right)} \\
= & \frac{2-2 \cos \theta_{1} \cos \theta_{2}}{2 \sin \theta_{1} \sin \theta_{2}} .
\end{aligned}
$$

Next, going to notations (18), we transform these formulas as

$$
\cosh \frac{S_{12}}{k}=\frac{\left(\xi_{1}-\xi_{2}\right)^{2}+\eta_{1}^{2}+\eta_{2}^{2}}{2 \eta_{1} \eta_{2}}
$$

Formula (23) is also true if the condition $\xi_{2}=\xi_{1}$ is met, then the formula (23) is reduced to formula (15). Relation between the formula for distance (23) and the cross- ratio

$$
\exp \left(\frac{1}{k}\left(\phi_{u}-\phi_{w}\right)\right)=\frac{u-x_{1}}{u-x_{2}} \frac{w-x_{2}}{w-x_{1}}
$$

is established, as shown in [4] and [5], on making use of the correspondence

$$
u=\frac{1}{\cos \theta} .
$$

In the Beltrami -Poincare model, geodesic lines on the Euclidean plane are represented either as straight lines perpendicular to the horizontal coordinate, or as semicircles with their ends lying on the abscissa axis. The integral of the potential corresponds to the distance on the vertical line, while the integral of the dipolar potential corresponds to the arc length of the semicircle with radius equal to the integration constant $\mathrm{R}$.

\section{REFERENCES}

[1] I. E. Tamm. Fundamentals of the theory of electricity. M. Nauka. GL.ed.Phys.Mat.lit.1989. ISBN 5-02-014244-1.

[2] E.Beltrami. "Teoria fondamentale degli spazi di curvature constante". Annali di Matematica Pura ed Applicata, ser II, 2 (1868) 232-255.

[3] N. A. Chernikov. The Lobachevsky plane, the Poincare model and the Bogolyubov transform in the theory of super-fluidity. Communications of JINR, Dubna. 1994, P2-94-469.

[4] R. M. Yamaleev. Hyperbolic cosine's and sine's theorems for the triangle formed by arcs of intersecting semicircles on Euclidean plane. Hindawi Publishing Corporation. J. Mathematics, Vol.2013, Article ID 920528, 2013. (dx.doi.org./10.1155/2013/920528)

[5] R.M. Yamaleev. Formulae for energy-momentum of relativistic particle regular at zero mass state. J.Mod.Phys. 2 (2011) 849-856 Relativistic dynamics. Phys.Atomic Nuclei 74 (2011) 1775-1782. 